\title{
Commentary on "The role of Chlamydia pneumoniae in the Etiology of Acne Rosacea: Response to the Use of Oral Azithromycin"
}

\author{
Adolfo C. Fernandez-Obregon \\ Hudson Dermatology and Skin Cancer Center, Hoboken, New Jersey, USA
}

\section{Article Info}

\section{Article Notes}

Received: February 13, 2019

Accepted: March 26, 2019

\section{*Correspondence:}

Adolfo C. Fernandez-Obregon, MD, FAAD, FACP, Hudson Dermatology and Skin Cancer Center, Hoboken, New Jersey, USA; Email: ob-1@att.net.

(c) 2019 Fernandez-Obregon AC. This article is distributed under the terms of the Creative Commons Attribution 4.0 International License.
It's been over a decade since we published the observation suggesting a possible role of Chlamydia pneumoniae in the etiology of acne rosacea. In that small patient series studied, the vast majority of subjects responded with moderate to marked improvement to a modified oral regimen of azithromycin. A significant number of the patients were identified with $C$. pneumoniae antigen in representative malar skin biopsies by immunoperoxidase technique using monoclonal antibody to $C$. pneumoniae. Nine of the 10 subjects received a modified regimen of azithromycin shown to be effective to treat acne rosacea. C. pneumoniae antigen was detected in malar biopsy specimens in 4 of 10 subjects by immunoperoxidase technique (using monoclonal antibody to $C$. pneumoniae. Serum antibodies against $C$. pneumoniae were detected in 8 of 10 intent-to-treat subjects. Using polymerase chain reaction, $C$. pneumoniae was not detected in peripheral blood mononuclear cells. The inflammatory response in tissues was characterized by a widespread infiltration of polymorphonuclear neutrophil cells, lymphocytes, and plasma cells, supporting the clinical diagnosis of acne rosacea. Nine of 10 subjects treated with azithromycin showed moderate to marked improvement of their acne rosacea. To our knowledge, this unique observation had not been reported elsewhere, and follow up studies including a study of the prevalence of antibodies to $C$. pneumoniae were never performed ${ }^{1}$.

Based on the results of this small study, it was tempting to suggest the presence of Chlamydia in the skin lesions of rosacea patients and their clinical improvement with azithromycin, would offer support to the notion Chlamydia infections could serve as one of the causes of acne rosacea. Azithromycin has been widely used in the treatment of Chlamydia infections, and its efficacy in the treatment of rosacea had already been documented ${ }^{2}$. It was never our intent to suggest a cause and effect relationship based on our findings linking C. pneumoniae in some way to the etiology of rosacea. At the time our study was performed there was excitement in the medical community at large trying to link C. pneumoniae to a host of medical conditions, including heart disease and other disorders associated with vascular inflammation, and hyperreactivity. It was hoped our observation would trigger interest in pursuing this issue beyond the limitations intrinsic to the small number of patients we studied, and the nominal immunological parameters evaluated. Gazing back now, though there was little interest expressed by sources of financial support, and others to probe deeper into our findings, other observations about the possible etiology of rosacea began to appear. 
Facial erythema may not only present clinically as a distinct entity as rosacea but can also be a symptom of other diseases. It is seen in common dermatoses such as eczema, psoriasis, acne and urticaria, as well as in rarer conditions such as disorders of keratinization, infectious diseases, porphyrias and neoplasia. Facial erythema may also present as a symptom of carcinoid syndrome, drug allergies, and cardiac disease or in rare cases as a feature of Bloom's syndrome, sarcoidosis, lymphoma, amyloidosis and other disease processes ${ }^{3}$. Besides the cases we reported linking C. pneumoniae to patients with rosacea, other associations have been introduced as probable in causing the red face symptoms recognized as acne rosacea, such as the presence of $H$. pylori and Demodex mite. Current knowledge suggests a potential link between $H$. pylori infection and skin diseases such as rosacea, chronic urticaria, hereditary or acquired angioedema due to C1esterase inhibitor deficiency, systemic sclerosis, SchönleinHenoch purpura, Sjögren's syndrome, Sweet's syndrome, and atopic dermatitis ${ }^{4}$. The bacterium has been implicated also in a number of extra-gastrointestinal disorders such as ischemic heart disease, ischemic cerebrovascular disease, and atherosclerosis, but a causal role for the bacterium is missing. "It would appear eradication of H. pylori would seem to be a beneficial impact on human health" ${ }^{5}$. Not surprising, some of the various drug regimens used to eradicate $H$. pylori also improve the appearance of rosacea, while the sequence of events by which these bacteria might cause these various conditions does not seem to follow Koch's postulates. Systematic studies examining the relationship between dermatologic entities and infection with $H$. pylori and documentation of the effect of $H$. pylori eradication are lacking ${ }^{6}$.

Of the trillions of human cells in the human body, approximately one in 10 is human, the rest are microbes. Their size is so small their existence hardly occupies any significant space in the body. The presence of microbes in the human body has created a study of the microbiome as it has become evident some fundamental microbes produce beneficial compounds like certain vitamins and protective anti-inflammatories that humans cannot produce themselves. Acquiring microbes is a lifelong activity beginning at birth. These microbes include bacteria, fungi, protozoa and other microorganisms. They can be found in all orifices and cavities of the human body as well as the skin. These organisms help the body against pathogenic microbes to prevent disease. Demodex mites form part of this defense and assist in ridding off dead skin cells and other waste from the skin. By doing so they help eliminate a large component of household dust ${ }^{7}$. Demodex folliculorum have often been found in 15 to 18 times greater numbers in rosacea patients than in healthy subjects. They are found in adults 18 years and older. Demodex folliculorum can be found in hair follicles, and neibomian glands of the eyelids, while $D$. brevis lives principally in the sebaceous glands of the $\operatorname{skin}^{8-10}$. A meta-analysis of 48 studies on Demodex found a significant association between the relative density of Demodex and the development of rosacea, suggesting its presence may be involved in the disease process ${ }^{11}$. Recent studies have demonstrated a correlation between Demodex infection and acne rosacea, and those having blepharitis were found to have a 2.5 -fold increase of Demodex infection compared to those without blepharitis ${ }^{12}$. Another fascinating connection is the observed presence of Bacillus oleronius, a bacterium on the Demodex mite that has been found to stimulate an immune response in some individuals with rosacea ${ }^{13}$. The $B$. oleronius association was found to hold true for those with ocular rosacea as well ${ }^{14}$. Looking at a possible relationship of Demodex density with inflammatory response and oxidative stress in rosacea, investigators found an association between an increased number of Demodex mites and changes in the facial microenvironment in rosacea. This increased Demodex density did not appear to alter disease severity, level of oxidative stress, or inflammation. Although none of the patients with rosacea had any underlying systemic disease, patients' systemic oxidative stress and inflammation parameters were found high in systemic circulation. Though not definitive, this study hints at the possibility patients with rosacea may be at risk for having other inflammatory systemic disease ${ }^{15}$. Recently, topical use of ivermectin has been found to be successful in treating acne rosacea, besides its anti-parasitic action aimed at Demodex, ivermectin has been shown to downregulate toll like receptor (TLR) activity, and its pro-inflammatory byproduct ${ }^{16}$. Further elucidation follows this discussion.

Other unusual relations have come to be recognized. Infectious foci, especially dental foci, seem to be rarely associated with the onset and progression of rosacea. Dermatologic treatments are determined by the severity of the disease, but eradication of infectious foci, can generate a significant improvement and may lead to a recovery. Low dose doxycycline, considered a sub-antimicrobial dose, has been used successfully to treat these infectious foci and periodontal disease as well ${ }^{17}$. Low dose doxycycline is also effective in the treatment of acne rosacea ${ }^{18}$.

The skin is challenged by microbes constantly, but it is rarely infected. As we have learned more about the protective homeostasis process that safeguards the body against disease, it is apparent factors exist to play a role in regulating or in failing to regulate disease activity. The alleged pathogens we recognize as potential causative agents, may well be innocent bystanders, vestige of an initiating process leading to disease, or perhaps even a secondary process of disease activity. Elucidation of this scheme is now ongoing in clinical research ${ }^{19}$.

Specific biomarkers that can be used to define rosacea 
have not been identified, though there are bioactive substances currently in vogue to present a reasonable hypothesis of what causes acne rosacea ${ }^{20}$. Patients with rosacea have been shown to have a high abundance of oligosaccharides in the tear fluid, which were not found in controls. This has the potential for the identification of glycomics that may serve as objective markers for the disease ${ }^{21}$.

A unifying hypothesis that addresses the etiology of rosacea, one that can accommodate these various observations, would have to start with the concept of a dysregulation of the innate immune response. As the first line of immunological defense in the body guarding against microbes, and environmental cellular damage, the innate immune response reacts in response to these hostile microbes and stimuli with the production of TLR, which are stimulated to release cytokines and antimicrobial molecules, including cathelicidin. These molecules have the ability to act as vasoactive and proinflammatory agents. In turn, they can promote leukocyte chemotaxis and angiogenesis. A local protease, kallikrein, is also produced, which leads to further production of cathelicidin.

Elevated expression of vascular endothelial and lymphatic growth factors, and vasoactive products released in this reactive response, leads to the vascular proliferation and increased permeability of the vessels. Factors that trigger flushing including emotional stress, hot beverages, spicy food, and very warm temperatures can worsen rosacea. Resolution of the erythema and flushing follows from the use of topical $\alpha 1$-adrenergic receptor agonist application by reducing the vascular hyperreactivity.

This cascade which results in further increasing cathelicidin leads to formation of reactive oxygen species (ROS) and matrix metalloproteinases (MMP). Factors that increase ROS and MMPs such as topical corticosteroids, and UVB light would naturally increase rosacea activity. Tetracyclines, and azithromycin act by reducing ROS and MMP levels, and this action improves rosacea ${ }^{22}$.

So, it appears, Chlamydia like H. pylori and Demodex may all play an interchangeable and partly innocent role in initiating and redefining a process that has been long programmed in our genes. Originally evolved to offer the first line of protection against noxious external stimuli, including pathogenic microbes, the innate immune response can also serve as a mechanism leading to the beginning of inflammatory disorders.

Cathelicidin dysfunction is now recognized to contribute to the development of cutaneous diseases such as atopic dermatitis and psoriasis through production of other additional substances responsible for the inflammatory cascade. It should be no surprise that novel therapies aimed to treat these disorders, are monoclonal antibodies and immunologically derived receptors targeting these pro-inflammatory disease-provoking agents. These new molecules, aimed to neutralize what we now recognized as products of the innate immune response, are man-made by-products derived from the adaptive immune response.

\section{References}

1. Fernandez-Obregon A, Patton DL. The role of Chlamydia pneumoniae in the etiology of acne rosacea: response to the use of oral azithromycin. Cutis. 2007 Feb; 79(2): 163-7.

2. Fernandez-Obregon A. Oral Use of Azithromycin for the Treatment of Acne Rosacea. Arch Dermatol. 2004 Apr; 140(4): 489-90.

3. Schuster Ch, Burg G. Das rote Gesicht [The red face]. Praxis (Bern 1994). 2004 Oct 13; 93(42): 1727-32.

4. Wedi B, Kapp A. Helicobacter pylori infection and skin diseases. J Physiol Pharmacol. 1999 Dec; 50(5): 753-76.)

5. Pakodi F, Abdel-Salam OM, Debreceni A, et al. Helicobacter pylori. One bacterium and a broad spectrum of human disease! An overview. J Physiol Paris. 2000 Mar-Apr; 94(2): 139-52.

6. Hernando-Harder AC, Booken N, Goerdt S, et al. Helicobacter pylori infection and dermatologic diseases. Eur J Dermatol. 2009 Sep-Oct; 19(5): 431-44. doi: 10.1684/ejd.2009.0739. Epub 2009 Jun 15.

7. Ackerman J. How bacterial affect our bodies protect our health. Scientific American. May 15 2012. http://www.scientificamerican. com/article.cfm?id=ultimate-social-network-bacteria-protectshealth

8. Jarmuda S, O’Reilly N, Zaba R, et al. Potential role of Demodex mites and bacteria in the induction of rosacea. J Med Microbiol. 2012; 61: 1504-1510.

9. Forton FMN. Papulopustular rosacea skin immunity and Demodex: pityriasis folliculorum as a missing link. J Eur Acad Dermatol Venereol. 2012; 26: 19-28.

10. Thoemmes MS, Fergus DJ, Urban J, et al. Ubiquity and diversity of human-associated Demodex mites. Public Library of Science One. 2014; 9(8): e106265. Doi:10.1371/journal.pone.0106265.

11. Zhao YE, Wu LP, Peng Y, et al. Retrospective analysis of association between Demodex infestation and rosacea. Arch Dermatol. 2010; 146(8): 896-902.

12. Sedzikowska A, Oseka A, Skopinski P. The impact of age, sex, blepharitis, rosacea, and rheumatoid arthritis on Demodex mite infection. Arch Med Sci. 2018 Mar; 14(2): 353-356.

13. Erbagci Z, Ozgoztaosi O. The significance of Demodex folliculorum density in rosacea. Int J Dermatol. 1998; 37: 421-425.

14. Li J, O’Reilly N, Sheha H, et al. Correlation between ocular Demodex infestation and serum immunoreactivity to Bacillus proteins in patients with facial rosacea. Ophthalmology. 2010; 117: 870-877.

15. Falay GT, Erdemir AV, Gurel MS, et al. The investigation of the rlationships of demodex density with inflmmatory response and oxidative stress in rosacea. Arch Dermatol Res. 2018 Nov; 310(9): 759-767.

16. Schaller M, Conser L, Belge K. Dual anti-inflammatory and antiparasitic action of topical ivermectin 1\% in papulopustular rosacea. J Eur Acad Dermatol Venereol. 2017 Nov; 31(11): 1907-1911.

17. Lesclous P, Maman L. An unusual case of a relationship between rosacea and dental foci. Oral Surg Oral Med Oral Pathol Oral Radiol Endod. 1999 Dec; 88(6): 679-82.

18. Korting HC, Schöllmann C. Current topical and systemic approaches to treatment of rosacea. J Eur Acade Dermatol Venereol. 2009 Aug; 23(8): 876-82. 
19. Schauber J, Gallo RL. J Allergy Clin Immunol. 2008 Aug; 122(2): 261-6. doi: 10.1016/j.jaci.2008.03.027. Epub 2008 Apr 25.

20. DiNardo A, Holmes AD, Muto $Y$, et al. Improved clinical outcome and biomarkers in adults with papulopustular rosacea treated with doxycycline and modified-release capsules in a randomized trial. J Am Acad Dermatol. 2016 Jun; 74(6): 1086-92.
21. An HJ, Ninonuevo M, Aguilan J, et al. Glycomic analyses of tear fluid for the diagnostic detection of ocular rosácea. J Proteome Res. 2005 Nov-Dec; 4(6): 1981-7.

22. Yamasaki K, Gallo RL. The molecular pathology of rosacea. J Dermatol Sci. 2009 Aug; 55(2): 77-81. 